\title{
УДК 551.462:551.463:551.464 \\ НОВЫЙ АКУСТИЧЕСКИЙ МЕТОД КОЛИЧЕСТВЕННОЙ ОЦЕНКИ ПУЗЫРЬКОВОГО ПОТОКА МЕТАНА В СИСТЕМЕ ДОННЫЕ ОТЛОЖЕНИЯ - ВОДНАЯ ТОЛЩА И ЕГО РЕАЛИЗАЦИЯ НА ПРИМЕРЕ МОРЯ ЛАПТЕВЫХ, СЕВЕРНЫЙ ЛЕДОВИТЫЙ ОКЕАН
}

\author{
Черных Денис Вячеславович ${ }^{12}$, \\ denis.chernykh.vl@gmail.com
}

Юсупов Владимир Исаакович, iouss@yandex.ru

Саломатин Александр Сергеевич', salomatin@poi.dvo.ru

Космач Денис Алексеевич ${ }^{1,2}$, den-kosmach@mail.ru

Константинов Антон Вячеславович4, toshic.konstantinov@gmail.com

Силионов Вячеслав Игоревич ${ }^{4}$, truexenox@gmail.com

Мазуров Алексей Карпович², akm@tpu.ru

\author{
Салюк Анатолий Назарович', \\ san@poi.dvo.ru
}

Шахова Наталья Евгеньевна², NataliaFletcher@yahoo.com

Орьян Густафсон Orjan.Gustafsson@aces.su.se

Колюбакин Андрей Анатольевич ${ }^{6.7}$, andrey.kolyubakin@gmail.com

Гершелис Елена Владимировна, elenapanova@tpu.ru

Лобковский Леопольд Исаевич ${ }^{8}$, llobkovsky@ocean.ru

Семилетов Игорь Петрович ${ }^{12}$, ipsemiletov@alaska.edu; igorsm@poi.dvo.ru

\footnotetext{
Тихоокеанский океанологический институт им. В.И. Ильичева, Россия, 690041, г. Владивосток, ул. Балтийская, 43.

2 Национальный исследовательский Томский политехнический университет, Россия, 634050, г. Томск, пр. Ленина, 30.

${ }^{3}$ Институт фотонных технологий ФНИЦ «Кристаллография и фотоника» РАН, Россия, 119333, г. Москва, Ленинский пр., 59.

${ }^{4}$ Дальневосточный федеральный университет Россия, 690091, г. Владивосток, ул. Суханова, 8.

${ }^{5}$ Центр исследований климата им. Б. Болина Стокгольмского университета, Швеция, 10691, Стокгольм, Сванте Аррениусвэг, 8 С

${ }^{6}$ Центр морских исследований МГУ им. М.В. Ломоносова Россия, 119991, г. Москва, Ленинские горы, 1.

7 ООО «Арктический научный центр», Россия, 119333, г. Москва, Ленинский пр., 155.

${ }^{8}$ Институт океанологии им. П.П. Ширшова РАН, Россия, 117997, г. Москва, Нахимовский пр., 36
}

Актуальность исследования обусловлена необходимостью разработки научно-обоснованного подхода к количественной оценке пузырькового переноса метана $\left(\mathrm{CH}_{4}\right)$ и других газов на основе акустических методов, позволяющих проводить достоверную оценку потока метана из областей его пузырьковой разгрузки с помощью эхолотов и гидролокаторов.

Цель исследования: разработка репрезентативного акустического метода количественной оценки потока метана из областей пузырьковой разгрузки в системе донные осадки - водная толща, основанного на определении количества всплывающих пузырьков, по данным о сечении их обратного рассеяния; обоснование репрезентативности разработанного метода путем сравнения с методом, основанным на проведении специальной калибровки научного эхолота по искусственному газовому факелу. объекты: газовые факелы - эманации газа в виде всплывающих со дна пузырьков, которые образуют в водной толще устойчивые области их повышенной концентрации.

Методы: разработанные авторским коллективом методы оценки потока $\mathrm{CH}_{4}$ из областей пузырьковой разгрузки, основанные на измерении: 1) сечения рассеяния всплывающих пузырьков; 2) калибровки по искусственному газовому факелу. 
Результаты. Представлен обзор современных акустических дистанционных методов, применяемых для оценки потоков СН 4 в водной толще, связанных с выходящими из дна и всплывающими пузырьками. На примере обширной области пузырьковой разгрузки $\mathrm{CH}_{4}$ на шельфе моря Лаптевых обоснована репрезентативность предложенного нового метода, основанного на расчете по сечению обратного рассеяния всплывающих пузырьков СН. Показано, что оценки величины пузырькового потока, полученные двумя методами: 1) новым методом, разработанным авторами, и 2) методом калибровки эхолота по искусственному га-

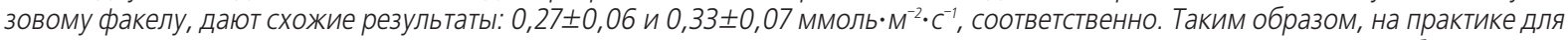
дистанционной и оперативной оценки потоков $\mathrm{CH}_{4}$ с участков его пузырьковой разгрузки можно использовать оба метода, с учетом занижения расчета потока по сечению обратного рассеяния примерно на 20 \% относительно реальных значений.

\section{Ключевые слова:}

Арктика, акустическая оценка, метан, газовые факелы, море Лаптевых, пузырьковый транспорт газа, поток метана.

\section{Введение}

Газовые факелы (ГФ), связанные с выходящими из донных отложений всплывающими пузырьками, зарегистрированы во многих районах Мирового океана [1-4], в том числе на арктическом шельфе (рис. 1) [5-10]. Наибольший интерес представляют мелководные ГФ, достигающие поверхности океана, значительная часть которых сосредоточена на арктическом шельфе [10]. Прямые измерения показали, что в большинстве случаев всплывающие из морского дна пузырьки состоят из метана $\left(\mathrm{CH}_{4}\right)[2,10]$. Предполагается, что формирование атмосферного максимума $\mathrm{CH}_{4}$ над $\mathrm{Ap \kappa -}$ тикой может быть связано с разгрузкой гигантских запасов гидратов, которые дестабилизируются вследствие деградации подводной мерзлоты [7-11]. Наиболее важную роль в этом процессе играет самый широкий и мелководный шельф Мирового океана - Восточно-Сибирский арктический шельф, на территории которого сосредоточена значительная доля арктических мелководных гидратов и более $80 \%$ которого содержат реликтовые субмаринные мёрзлые толщи $[11,12]$, состояние которых определяет масштабы выбросов пузырькового $\mathrm{CH}_{4}$ из донных осадков в водную толщу и атмосферу [6-10]. Более того, на экспериментальном и модельном уровне доказано [8, 13-15], что скорости их деградации в морях Восточной Аркти- ки многократно превосходят принятые IPCC в 2014 г. оценки. Поэтому прогрессирующая деградация подводных мерзлых толщ морей Восточной Арктики может привести к массированному выбросу гидратного метана в водную тощу и атмосферу, что может привести к труднопредсказуемым последствиям $[11,15,16]$.

Пузырьковый транспорт - наиболее эффективный механизм доставки метана из донных осадков в водную толщу и атмосферу $[3,4,10]$, так как позволяет избежать окисления в зоне сульфат-редукции. Метан является эффективным парниковым газом, вклад которого в глобальное потепление неуклонно растет [17]. Поэтому актуальным является развитие методов, позволяющих дистанционно и оперативно проводить количественную оценку пузырькового потока метана в водную толщу и атмосферу на обширных участках акватории Мирового океана.

Акустические методы, основанные на генерации акустического импульса и приеме рассеянного акустического сигнала, являются эффективными для наблюдения за всплывающими пузырьками и получения оценок количества метана, доставляемого ими в воду и атмосферу. Кроме того, данные методы позволяют дистанционно, с помощью штатных или специализированных судовых эхолотов и гидролокаторов, обнаружить всплывающие пу-
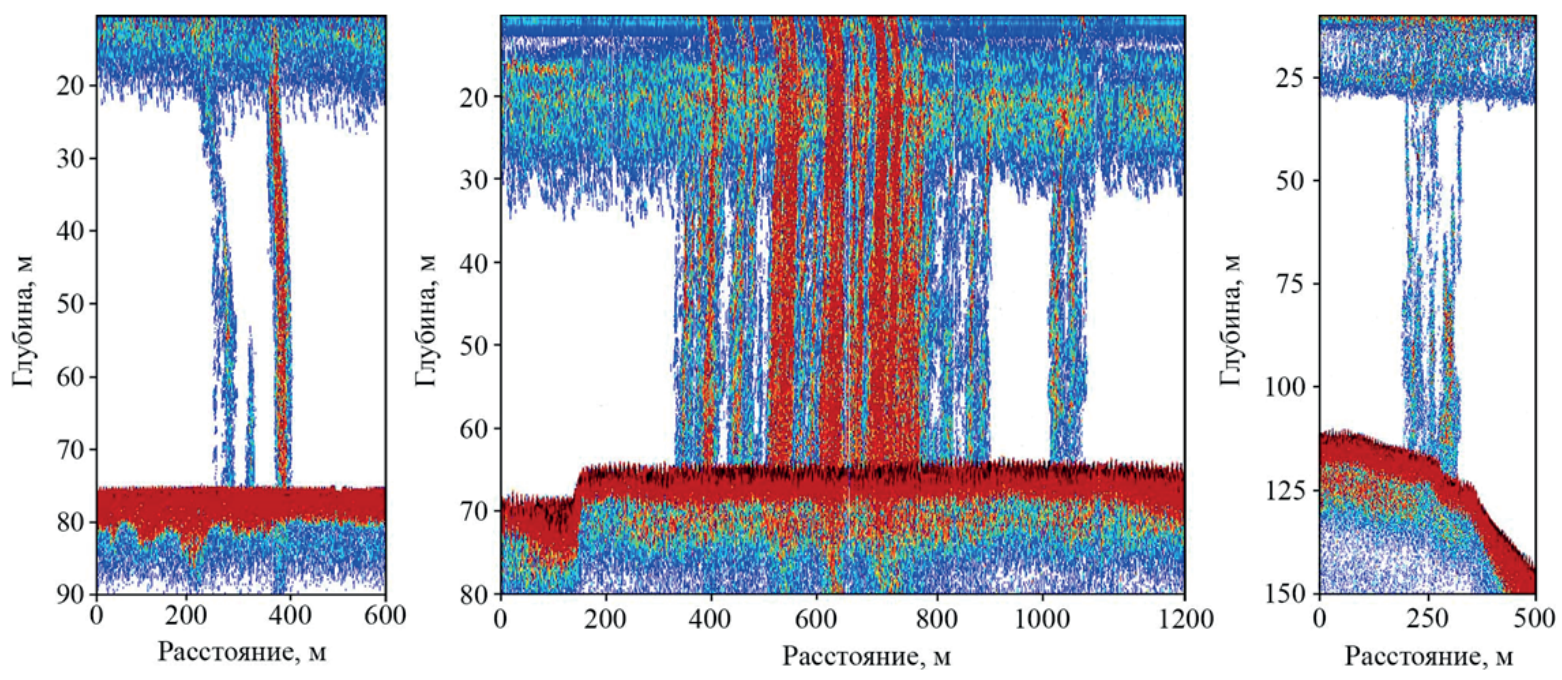

Рис. 1. Примеры эхорамм газовых факелов, зарегистрированных на арктическом шельфе в период с 2011 по 2016 гг.

Fig. 1. Examples of hydro-acoustical images of detected seep fields at Arctic shelf from 2011 to 2016 
зырьки миллиметрового размера на расстоянии до нескольких сотен метров и более, оценить их размеры и скорости всплытия [2, 4-6, 10, 18-25].

В данной работе рассматриваются различные методы акустической оценки количества $\mathrm{CH}_{4}$, доставляемого всплывающими пузырьками, и их сравнение. Показана возможность использования метода измерения сечения обратного рассеяния от всплывающих пузырьков для количественной оценки пузырькового переноса в системе «дно водная толща». В качестве примера приводятся сравнительные оценки величины пузырькового потока из обширной области пузырьковой разгрузки метана на шельфе моря Лаптевых, рассчитанные модифицированным методом измерения сечения обратного рассеяния, и с помощью калибровки эхолота по искусственному ГФ, выполненному авторским коллективом ранее [11].

\section{Акустические методы оценки потока метана}

Под ГФ мы будем понимать газовые эманации в виде всплывающих со дна пузырьков, которые образуют в водной толще устойчивые области их повышенной концентрации. Следует различать мелководные ГФ, как правило, расположенные на шельфе [4, 8-10, 17], и глубоководные ГФ [4, 20]. На дне каждый мелководный ГФ зачастую занимает обширную область нередко с площадью в несколько квадратных километров [10]. В отличие от этого поперечные размеры глубоководных ГФ, как правило, меньше 10 м («точечные» ГФ), и они часто расположены обособлено друг от друга $[4,20]$. Для случая «точечных» и обособленных источников ГФ, когда источники хорошо разрешаются эхолотом (обычно это соответсвует «глубоководному» ГФ), будем говорить о потоке $F$, под которым будем понимать количество метана, переносимого всплывающими в ГФ пузырьками через горизонтальную поверхность в единицу времени. В случае ГФ, источники которых расположены достаточно плотно, когда они не разрешаются эхолотом (обычно это «мелководные» ГФ), будем говорить о потоке $F s$ с единицы площади, под которым будем понимать количество метана, переносимого всплывающими в водной толще пузырьками через горизонтальную поверхность единичной площади в единицу времени.

Расчет потока метана, переносимого пузырьковым транспортом, по акустическим данным является сложной многофакторной задачей, плохо поддающейся моделированию в лабораторных условиях. Для решения этой задачи в настоящее время применяется синтез теоретических расчетов, лабораторных исследований и натурных экспериментов $[4,10,21,25-29]$.

Рассмотрим некоторые методы акустической дистанционной оценки потока метана в областях его пузырьковой разгрузки, основанные на использовании установленных на судах однолучевых эхолотов. В этом случае реализуется методика дистанционного акустического моностатического зондирования, когда излучатель, являясь одновременно приемником, излучает в водную толщу в направлении морского дна импульсные сигналы, а источником информации являются принятые приемником акустические сигналы, рассеянные в обратном направлении. При такой схеме способность любого физического объекта, в том числе и пузырьков, рассеивать звук в направлении к приемнику характеризуется его сечением обратного рассеяния [29], имеющего размерность площади:

$$
\sigma_{b s}=\frac{I_{b s} L^{2}}{I}
$$

где $I_{b s}$ - интенсивность рассеянного сигнала в точке приема; $L$ - расстояние от точки излучения-приема до объекта; I - интенсивность падающей на объект волны. В литературе часто используется понятие «сила цели» $T S$, характеризующей сечение обратного рассеяния, выраженное в децибелах [30, 31]:

$$
T S=10 \lg \frac{\sigma_{b s}}{A_{1}},
$$

где $A_{1}=1 \mathrm{~m}^{2}$ - единичная площадь.

При дистанционном акустическом исследовании областей пузырьковой разгрузки полезно различать два случая: 1) отдельные пузырьки акустически разрешаются и на эхограмме каждый из них виден в отдельности; 2) отдельные пузырьки акустически не разрешаются.

Если удается проследить за отдельными пузырьками, акустические методы позволяют прямо определить скорости всплытия, размеры отдельных пузырьков и оценить поток метана в водную толщу $[4,24,29,32-36]$. Количество молей газа $F_{s}$, переносимого всплывающими пузырьками через горизонтальную поверхность единичной площади в единицу времени, в этом случае будет определяться с помощью выражения:

$$
F_{s}=\frac{1}{V_{m} t S} \sum_{i=1}^{N} v_{i},
$$

где $V_{m}$ - молярный объем метана при температуре и давлении на данном горизонте; $S$ - площадь озвученной зоны на данном горизонте; $N$ - число пузырьков, пересекающих за время наблюдения $t$ данную поверхность; $v_{i}$ - объем $i$-го пузырька [4] или

$$
F_{s}=\frac{\left\langle V_{b}(r)\right\rangle P(h) N_{b}}{R T t S} .
$$

Здесь $V_{b}(r)$ - объем пузырька с радиусом $r$ (без учета его несферичности); $\langle>$ - оператор усреднения; $P(h)$ - давление на глубине $h ; N_{b}$ - число обнаруженных пузырьков; $t$ - длительность измерений; $R$ - газовая постоянная; $T$ - температура, ${ }^{\circ} \mathrm{K}$ [26]. Площадь озвученной зоны на данном горизонте $L$ определяется как:

$$
S=L^{2} \Psi_{D},
$$

где $\Psi_{D}$ - интегральная ширина диаграммы направленности эхолота [30]: 


$$
\Psi_{D}=\oint_{4 \pi} D^{2} d \grave{U}
$$

где $D$ - амплитуда диаграммы направленности преобразователя; $d \Omega$ - приращение телесного угла.

Недостатком данного метода является необходимость определения размеров всплывающих пузырьков. Если применяются обыкновенные однолучевые эхолоты, то невозможно определить местоположение пузырька в диаграмме направленности антенны эхолота, что приводит к большим ошибкам. Кроме того, если распределение пузырьков по размерам широкое, то появляется неоднозначность в определении размера пузырька, связанная с тем, что одному и тому же уровню рассеяния соответствуют пузырьки разных размеров. Эту трудность можно обойти, если применять многочастотные гидроакустические системы. Но такое решение лежит вне области данного исследования.

Полезны в этом случае эхолоты с двойным лучом, которые позволяют существенно повысить точность определения сечения обратного рассеяния цели [37]. Конструктивная особенность таких эхолотов заключается в использовании двух соосных лучей с сильно различающимися ширинами диаграмм направленности. В расчет включаются только те рассеиватели, которые регистрируются одновременно и в широком, и в узком лучах. В этом случае цель находится в центре широкого луча, по которому и производится расчет. Наиболее эффективными являются эхолоты с расщепленным лучом, которые позволяют определить местоположение цели в луче за счет более сложной конструкции приемо-излучающей антенны и дополнительных фазовых измерений [20-22, 26].

Для уточнения размеров всплывающих пузырьков можно воспользоваться оптическими данными, но можно оценить размер пузырька и по его скорости всплытия. Это можно сделать, например, воспользовавшись графическими или эмпирическими зависимостями скорости всплытия пузырька от его размера $[32,38]$. Следует учитывать, что скорости всплытия крупных пузырьков, с радиусом более 1 мм, слабо зависят от размеров, поэтому точность подобных оценок невелика [32, 38]. Опубликована также подпрограмма в среде Matlab, которая позволяет рассчитать скорости всплытия пузырьков по их размерам [23]. Замечательно, что в случае, когда различаются отдельные пузырьки, скорости всплытия можно оценить непосредственно по акустическим данным [4, 32, 38]. В этих случаях при малых скоростях движения судна каждый пузырек отображается на эхограмме в виде наклонной линии. Средняя скорость всплытия пузырька $V$ легко определяется по наклону этой линии [4]:

$$
V=\frac{\cos (\varphi) \Delta h}{\Delta t},
$$

где $\Delta h$ - разница глубин видимых концов траектории пузырька на эхограмме; $\Delta t$ - соответствую- щий интервал времени; $\varphi$ - полуширина диаграммы направленности по уровню, равному отношению амплитуд сигналов на концах траектории к максимальному сигналу от пузырька. При хорошем соотношении сигнал/шум этот угол близок к углу нуля диаграммы направленности, который для типичных әхолотов практически совпадает с шириной диаграммы направленности по уровню 0,7. Данное выражение справедливо, если за время наблюдения размеры пузырька меняются незначительно. Далее из зависимости скорости всплытия пузырьков от размеров [26, 32, 38, 39] можно для каждого $i$-го пузырька оценить его эквивалентный сферический радиус $r_{i}$ и, следовательно, его объем $v_{i}$. Оценить размеры пузырьков можно и исходя из наблюдаемых максимальных высот ГФ [4, 28], причем в качестве высоты ГФ принимается не та высота, которую достигают отдельные пузырьки, а высота, где ГФ на эхограмме еще прослеживается в виде непрерывной области повышенного рассеяния.

Если отдельные пузырьки в ГФ не различаются, но распределения пузырьков по размерам, скоростям всплытия и форме известны, то поток метана может быть оценен по сечению объемного обратного рассеяния звука, обусловленного рассеянием на всплывающих пузырьках $[4,10,25]$. Основополагающим в этой оценке является положение, что в приближении однократного рассеяния, сечение рассеяния от объема с пузырьками равно сумме сечений отдельных пузырьков [40]. Таким образом, в приближении однократного рассеяния сечение обратного рассеяния $\sigma_{b s}$ от объема с пузырьками, одновременно участвующем в рассеянии, может быть вычислено по формуле [30]:

$$
\sigma_{b s}=N_{0} \int_{0}^{\infty} \sigma_{b s r} n(r) d r,
$$

где $r$ - радиус пузырька; $N_{0}$ - количество пузырьков в исследуемом объеме; $n(r)$ - функция плотности распределения пузырьков по размерам; $\sigma_{b s r}-$ сечение обратного рассеяния одиночного пузырька радиуса $r$. Для случая «глубоководных» ГФ или ГФ от «точечного» и обособленного источника $N_{0}$ определяется количеством пузырьков в ГФ в диапазоне глубин $h \pm l / 4$, где $h$ - глубина, $l$ - длина зондирующего импульса. В случае «мелководных» ГФ или ГФ, источники которых расположены достаточно плотно и не разрешаются эхолотом, $N_{0}$ определяется количеством пузырьков в ГФ в сегменте телесного угла $\Psi_{D}$ интегральной ширины диаграммы направленности эхолота в диапазоне расстояний от $h \pm l / 4$ от излучателя.

Следует учитывать, что в случаях мощных ГФ, когда расстояния между пузырьками становятся сопоставимыми с их радиусом, они начинают влиять друг на друга [41]. Такое взаимодействие между близко расположенными пузырьками может привести к значительному уменьшению их сечения обратного рассеяния. Поэтому в случаях, когда в ГФ расстояние между пузырьками стано- 
вится сопоставимым с их радиусом, акустические оценки потока по сечению обратного рассеяния дадут заниженные значения.

Переносимый пузырьками поток газа (число молей газа в секунду) $F$ связан с функциями плотности распределения пузырьков по размерам $n(r)$ и форме $\Phi(r)$ очевидным выражением [4, 28]:

$$
F=\frac{N_{0}}{V_{M}} \frac{4 \pi}{3} \int_{0}^{\infty} \Phi(r) V(r) r^{3} n(r) d r,
$$

где $V(r)$ - скорость всплытия пузырьков.

Исключая $N_{0}$ из выражений (1) и (2) можно легко получить следующую зависимость между потоком и сечением обратного рассеяния [26, 36,37$]$ :

$$
F=\frac{1}{V_{M}} \frac{4 \pi}{3} \frac{\int_{0}^{\infty} \Phi(r) V(r) r^{3} n(r) d r}{\int_{0}^{\infty} \sigma_{b s r} n(r) d r} \sigma_{b s}=K \frac{\sigma_{b s}}{V_{M}},
$$

где $K$ - сложная функция большого количества параметров, таких как функции распределения пузырьков по размерам, форме, скоростям всплытия, частоты зондирования, состояния поверхности пузырьков, глубины, акустических свойств пузырьков и др. Выражение (3) определяет поток метана по измеренному сечению обратного рассеяния для случая "глубоководных» ГФ или ГФ от «точечного» и обособленного источника. В случаях, когда источников много и они не разрешаются эхолотом, выражение (3) для потока с единицы площади принимает вид:

$$
F_{s}=K \frac{\sigma_{b s}}{V_{m} S}
$$

где $S$ - эффективная озвученная площадь.

При известных распределениях пузырьков по размерам, форме, скоростям всплытия можно рассчитать функцию $K$ и использовать выражение (3) для расчета потока, однако в большинстве случаев такую информацию получить практически невозможно. Поэтому ряд исследователей для оценки потока использует упрощенные методы расчета, средние значения скорости всплытия, размеров, а форму пузырьков принимают за сферическую.

Так, например, в работе [4] в предположении, что все пузырьки имеют один и тот же радиус $r$ и всплывают с постоянной скоростью $V$, радиусы пузырьков больше резонансного, а $k r<<1$, где $k$ - волновое число, для потока метана (в молях в секунду) получено выражение:

$$
F=\frac{8 \pi r}{3 V_{M} l} V \sigma_{b s},
$$

где $V_{M}$ - молярный объем метана при температуре и давлении на исследуемом горизонте; $l$ - длина зондирующего импульса.

Другие авторы используют средние значения для одних параметров и различные аппроксимирующие зависимости для других. Так, например, в работе [26] использована экспоненциальная аппроксимация распределения пузырьков по разме- рам со скачкообразным уменьшением до нуля при $r \leq r_{c}$, где $r_{c}$ - радиус, меньше которого пузырьки не наблюдаются: $n(r)=e^{-a r}$ при $r \geq r_{c}$ и $n(r)=0$ при $r \leq r_{c}$. Из лабораторных и натурных исследований было получено, что $r_{c}$ колеблется в пределах $0,5-1 \mathrm{Mм}, \mathrm{a}$ параметр $\alpha=1,1 \mathrm{~mm}^{-1}$.

Стоит отметить, что предложенные выше методы определения радиусов выплывающих пузырьков имеют оценочный характер. Для определения распределения пузырьков по размерам, форме, скоростям всплытия необходимо привлекать другие методы, например, оптические, которые позволяют непосредственно измерить размер каждого всплывающего из морского дна пузырька, определить его форму и скорость всплытия [10].

Во всех приведенных выше примерах оценки потока метана, переносимого всплывающими пузырьками, для оценки сечения обратного рассеяния одиночного пузырька $\sigma_{b s r}$ использовалось выражение:

$$
\sigma_{b s r}=\frac{r^{2}}{\left(\left(\frac{r_{r e s}}{r}\right)^{2}-1\right)^{2}+\delta^{2}},
$$

где $r_{\text {res }}$ - резонансный радиус пузырька на рабочей частоте эхолота; $\delta$ - постоянная затухания пузырька. Аналогичные подходы при оценке потока, переносимого всплывающими пузырьками, по данным акустического зондирования использует подавляющее число авторов $[4,9,26,29]$. Выражение (4) справедливо при условии $k r<<1$, что вносит ограничение на выбор частоты эхолота. Рабочая частота 38 кГц эхолотов, используемых для оценок потока метана многими авторами [20, 26 , 42-45], является, строго говоря, слишком высокой, так как на этой частоте $k r=1$ уже для пузырьков радиуса 6 мм. Однако в работе [28] показано, что, с учетом реальной несферичности всплывающих пузырьков, область применения уравнения (5), с приемлемой точностью можно продлить в область $k r>1$. С другой стороны, как видно из уравнения (5), сечение рассеяния пузырька, радиус которого меньше, чем резонансный, уменьшается с уменьшением радиуса пузырька как $r^{6}$, то есть пузырьки радиус которых меньше резонансного практически не участвуют в формировании сигнала обратного рассеяния. Это ограничение особенно существенно для глубоководных источников. Резонансные размеры пузырьков определяются с помощью выражения [28]:

$$
r_{\text {res }}=\frac{1}{2 \pi f_{0}} \sqrt{\frac{3 \gamma\left(P_{0}+\rho_{w} g h\right)}{\rho_{W}}},
$$

где $f_{0}$ - частота излучения эхолота; $\gamma$ - показатель адиабаты для газа в пузырьке; $P_{0}$ - атмосферное давление; $\rho_{w}$ - плотность воды; $g$ - ускорение силы тяжести; $h$ - глубина.

Из выражения (6) следует, что если на глубине 50 м для широко используемых частот эхолотов 12 и 18 кГц резонансные радиусы пузырьков со- 
ставляют 0,6 и 0,4 мм, то на глубине 1 км они возрастают до 2,6 и 1,6 мм, соответственно.

Для оценок потока метана из мелководных источников всплывающих пузырьков можно подобрать частоту, которая удовлетворяет с одной стороны условию $k r<<1$, и, с другой стороны, условию $r_{c} \geq r_{\text {res. }}$. Исходя из размеров реально наблюдаемых пузырьков [9, 43], частотой, которая удовлетворяет этим двум условиям, является частота 18 кГц. Однако для проведения оценок потока газа из областей пузырьковой разгрузки можно использовать и другие частоты, в том числе в диапазоне нескольких сотен килогерц. Неоспоримым преимуществом таких высокочастотных систем является их небольшие размеры, мобильность и низкое энергопотребление. Это делает их удобными для проведения исследовательских работ с использованием различных, в том числе специально необорудованных, судов.

Что касается глубоководных ГФ, то для них подобрать нужную рабочую частоту эхолота более затруднительно, поскольку с глубиной сужается диапазон, на котором одновременно удовлетворяются неравенства $r_{c} \geq r_{\text {res }}$ и $k_{r}<<1$. Эти обстоятельства могут приводить к появлению больших ошибок при оценке потока метана акустическим способом.

Следует учитывать, что для мелководных областей пузырьковой разгрузки метана возникают дополнительные трудности, поскольку на малых глубинах начинают сильно влиять резонансные эффекты, так как с уменьшением давления добротность пузырьков $Q=1 / \delta$ сильно возрастает. Для больших глубин (порядка 1000 м) добротность пузырька в основном ограничивается сверху теоретическим пределом, связанным с переизлучением, и не может превышать 7. Для глубин в несколько десятков метров добротность значительно увеличивается и может достигать нескольких десятков [46]. Это приводит к тому, что на малых глубинах влияние резонансных эффектов значительно возрастает и сечение одного резонансного пузырька может быть больше, чем нескольких нерезонансных.

Достаточно простые выражения для расчета потока метана по данным акустического зондирования основаны, во-первых, на расчете сечения обратного рассеяния одиночного пузырька с помощью выражения (5) и, во-вторых, на упрощенных аппроксимациях или использовании средних значений вместо реальных распределений таких величин, как радиус пузырька, его объем, скорость всплытия. Такое упрощение не всегда обосновано и может привести к большим ошибкам. Также во многих случаях, особенно в случае глубокого моря, не удается подобрать рабочую частоту эхолота, чтобы выполнялись неравенства $k_{r}<<1$ и $r_{c} \geq r_{\text {res }}$. В случае мелководных ГФ большие ошибки могут возникнуть из-за маленькой точности в определении добротности пузырьков. Кроме того, ошибки в оценке потока метана могут быть связаны с тем, что не учитывается распределение пузырьков по форме и используются приближенные значения коэффициента затухания акустических волн. Поэтому представляет большой практический интерес для получения экспресс оценок экспериментально определять в условиях, максимально приближенных к реальным, коэффициент $K$ в выражении (3).

Поскольку этот коэффициент является сложной функцией большого количества параметров, которые в большинстве случаев не известны, представляют интерес методы, основанные не на рассчете этого коэффициента, а на его экспериментальном определении $[10,21]$.

Для получения оценочных значений потока метана из морского дна в водную толщу использовался метод, аналогичный изложенному в работе [21]. Основным преимуществом такого подхода является то, что для проведения оценки потока газа не требуется знания распределений пузырьков по размерам, форме, скоростям всплытия и гидроакустических характеристик эхолота. Основное отличие метода [10] от метода, предложенного в [21], состояло в том, что калибровка производилась не в лабораторных условиях, а в натурных условиях, максимально приближенных к условиям исследуемого района. Кроме того, акустические сигналы излучались и принимались не в горизонтальном, как в работе [21], а в вертикальном направлении. Выбор такой конфигурации калибровки связан с тем, что измерения потока метана проводились с помощью установленных на борту судна эхолотов и зондирование производилось в направлении дна, то есть в вертикальном направлении. Измерения проводились так же, как и в [21], в моностатической моде, когда акустические сигналы излучаются и принимаются одним и тем же акустическим преобразователем эхолота. При акустическом исследовании с поверхности моря излучатель эхолота погружается на некоторую глубину (обычно порядка нескольких метров), поэтому расстояние от излучателя до рассеивающего объема $L$ несколько отличается от глубины этого объема $h$. На глубине $h$ сигнал обратного рассеяния формируют всплывающие пузырьки, находящиеся в эффективном рассеивающем объеме $\Delta V$, который для $h>>c \tau / 2$ можно оценить как [30]:

$$
\Delta V \approx \frac{c \tau}{2} L^{2} \Psi_{D}
$$

где $c$ - скорость звука; $\tau$ - длительность импульса; $\Psi_{D}$ - интегральная ширина диаграммы направленности эхолота. Связь регистрируемой эхолотом силы цели от всплывающих пузырьков в этом эффективном рассеивающем объеме $\Delta V$ описывается стандартным уравнением гидролокации [31]:

$$
T S=R L-S L-T L,
$$

где $T S$ - сила цели пузырьков в эффективном рассеивающем объеме $\Delta V ; R L$ - уровень принимаемого сигнала в дБ/мПа; $S L$ - уровень излучения в дБ/мПа на расстоянии 1 м; $T L$ - потери сигнала, обусловленные сферическим расхождением и поглощением: 


$$
T L=2\left(20 \log \left(\frac{L}{L_{1}}\right)+a L\right)
$$

где $L_{1}=1 \mathrm{~m} ; \alpha-$ коэффициент затухания в морской воде в дБ/м.

Значение для $R L$ вычисляется из следующего выражения:

$$
R L=10 \log \left(U_{n}^{2}\right)+K_{1},
$$

где $U_{u}^{2}$ - приведенная амплитуды принятого сигнал для каждого импульса излучения $n$; $K_{1}-$ коэффициент, зависящий от параметров эхолота.

Обычно в современных эхолотах предусмотрена компенсация потерь, обусловленных сферическим расхождением и поглощением. В этом случае $T L$ из уравнения (7) исключается. Поскольку $S L$ постоянная величина, то:

$$
T S=10 \log \left(U_{0}^{2}\right)+K_{2},
$$

где $K_{2}$ - калибровочный коэффициент; $U_{0}^{2}$ - приведенная амплитуда сигнала для каждого импульса излучения $n$ при компенсации потерь, обусловленных сферическим расхождением и поглощением. С учетом этого для потока ГФ от одиночных, локализованных ГФ:

$$
F=K_{B} \frac{U_{0}^{2}}{V_{M}} .
$$

В случае «мелководных» факелов, когда источники акустически не разрешаются, определяется поток с единицы площади:

$$
F=K_{B} \frac{U_{0}^{2}}{V_{M} S},
$$

где $S$ - эффективная рассеивающая площадь.

Для применения метода в случае глубоководного ГФ, как следует из выражения (8), достаточно провести простые калибровочные измерения зависимости уровня обратного рассеяния от всплывающих пузырьков от величины потока газа. В случае «мелководного» ГФ, когда отдельные источники не разрешаются, помимо таких калибровочных измерений необходимо знать угловые характеристики системы для определения необходимой по выражению (9) величины эффективной рассеивающей площади $S$. Она определяется по расстоянию от излучателя эхолота до исследуемого объема и по интегральной ширине диаграммы направленности направленности эхолота $\Psi_{D}$. $K$ сожалению, $\Psi_{D}$ в технических паспортах эхолотов не приводится. Для поршневого излучателя она может быть оценена с помощью простого выражения [30]:

$$
\Psi_{D}=\frac{5,48}{(k r)^{2}},
$$

где $k$ - волновое число; $r$ - радиус излучателя эхолота.

\section{Результаты натурных измерений}

Основным условием успешного применения эхолотов для регистрации пузырьков является то, что их рабочая частота $f$ должна быть, с одной стороны, выше резонансной частоты всплывающих пузырьков $f_{r}$, поскольку при частоте зондирования меньшей $f_{r}$ эффективность регистрации резко падает. С другой стороны, $f$ должна быть достаточно низкой, чтобы уменьшить затухание при распространении акустического сигнала от излучателя до рассеивающего объема и обратно. В работе для обнаружения и исследования областей пузырьковой разгрузки использовался однолучевой научный эхолот Simrad EK15 с рабочей частотой 200 кГц, которая лежит выше резонансных частот всплывающих пузырьков для всех выбранных диапазонов глубин от 0 до $100 \mathrm{M}$.

В соответствии с поставленными в работе целями, на шельфовом полигоне были выполнены: 1) стандартная калибровка эхолота по калибровочной цели; 2) специальная калибровка по искусственному ГФ. Для калибровки эхолота применялась карбид-вольфрамовая калибровочная цель диаметром 38,1 мм (рис. 2).

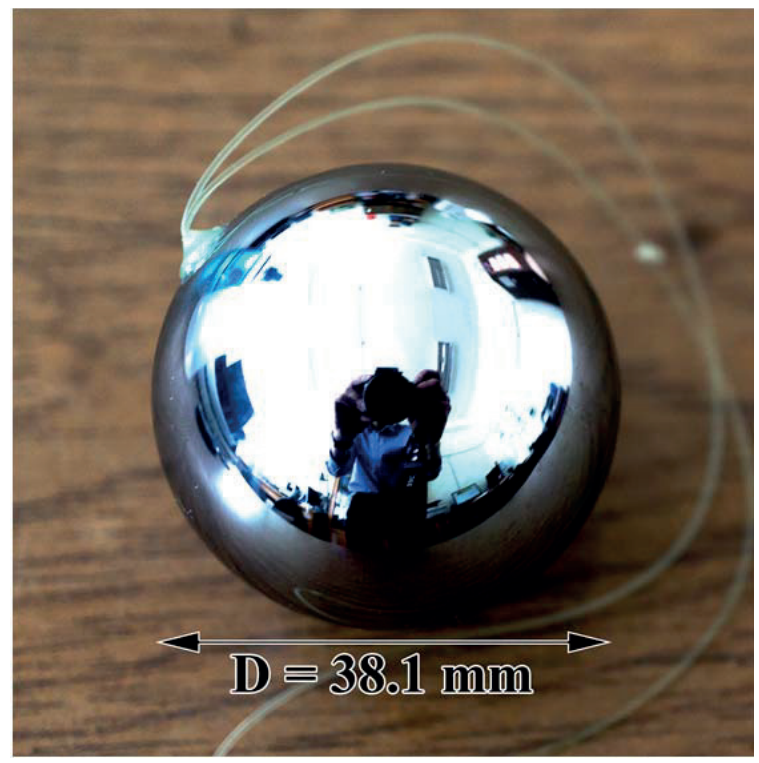

Рис. 2. Карбид-вольфрамовая калибровочная сфера

Fig. 2. Tungsten carbide calibration sphera

Обработка данных производилась с помощью пакета прикладных программ для решения задач технических вычислений MatLab. При обработке и представлении массива данных определялись средние величины и стандартные отклонения. Существование различий между отдельными выборками проверяли с помощью критерия Стьюдента $p<0,05$.

Во время проведения стандартной калибровки цель опускалась с борта судна на глубину $4,5 \mathrm{~m}$, помещалась в середину озвученной зоны и для установленных рабочих параметров әхолота (частота, мощность, длительность импульса и др.) определялась максимальная величина квадрата амплитуды принятого сигнала на выходе эхолота $U_{0}^{2}$, при этом $U_{0}^{2}-$ приведенная амплитуда сигнала 

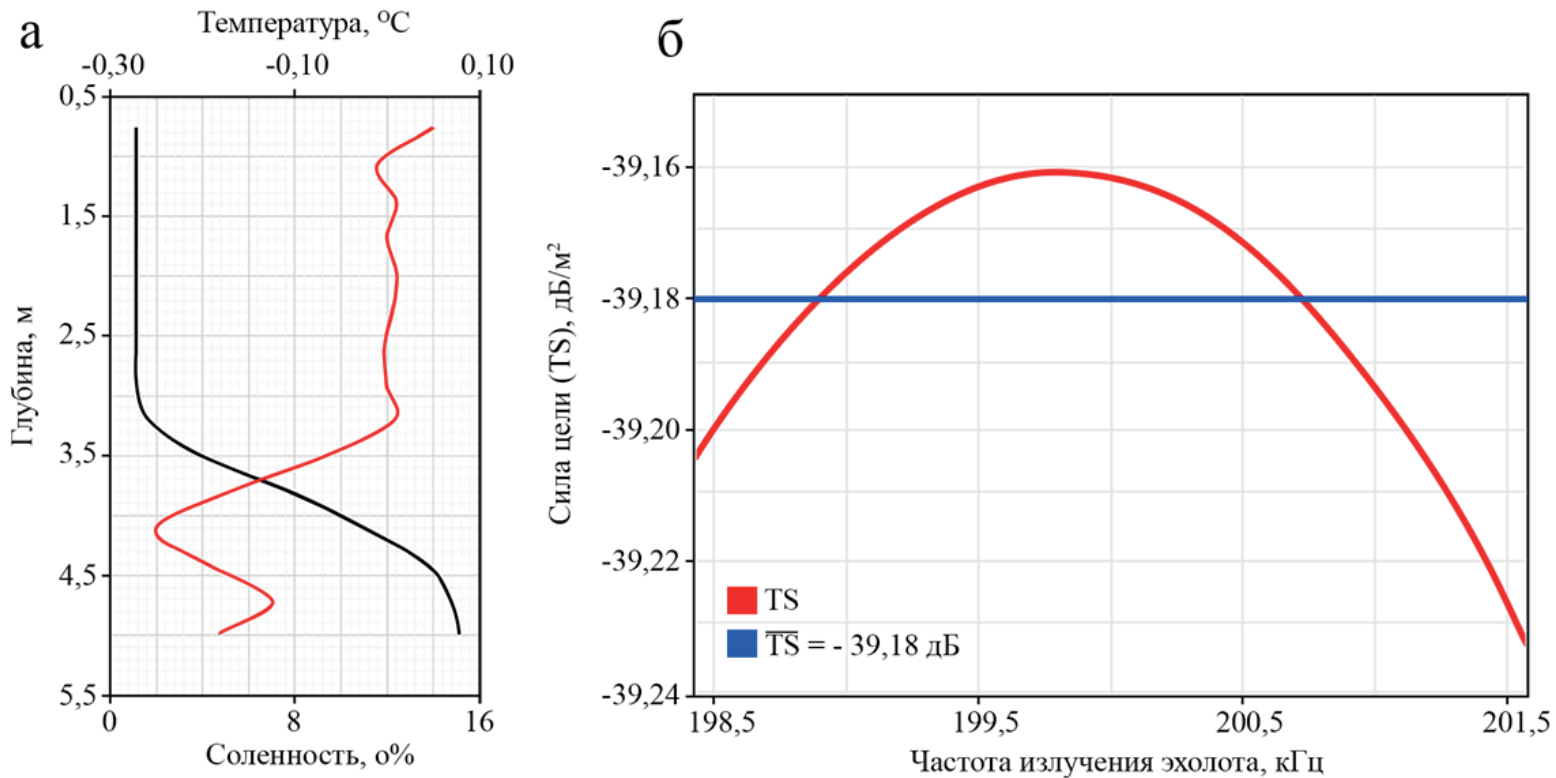

Рис. 3. Профили температуры и солености (а), полученные во время калибровки научного эхолота Simrad ЕК15, и калибровочный график зависимости «силы цели» от рабочей частоты эхолота (б, красная линия). Синей линией на рис. б показано среднее значение TS

Fig. 3. Profiles of temperature and salinity (a) got during echosounder Simrad EK15 calibration; calibration curve of dependence of target strength (TS) on echosounder frequency $(6$, red line). Blue line illustrates an average value of TS

для каждого импульса излучения с учетом компенсации потерь, обусловленных сферическим расхождением и поглощением. При компенсации учитываются реальные данные о распределении температуры, солености и скорости звука в водной толще в данном месте. Гидрофизические данные были получены с помощью гидрологического 30 - $^{-}$ да CastAway-CTD с рабочими глубинами до 100 м, частотой квантования 5 Гц, точностью измерения солености $\pm 0,1$ PSU и температуры $\pm 0,05^{\circ} \mathrm{C}$. Определение конкретного значения $T S$ калибровочной цели производилось согласно [47-49] по значениям температуры и солёности на глубине цели в момент калибровки (рис. $3, a)$.

Из калибровочной зависимости от частоты излучения научного эхолота Simrad EK15 (рис. 3, б) следует, что среднее значение $T S$ составляет $-39,18 \mathrm{~dB} / \mathrm{m}^{2}$, что соответствует сечению обратного рассеяния калибровочной сферы $\sigma_{b s 0}=1,21 \mathrm{~cm}^{2}$.

В результате проведенной калибровки по калибровочной цели был определен коэффициент пропорциональности $K_{0}$ между сечением обратного рассеяния калибровочной цели $\sigma_{b s 0}$ и квадратом приведенной амплитуды сигнала на выходе эхолота при калибровке:

$$
K_{0}=\frac{\sigma_{b s 0}}{U_{0}^{2}} .
$$

Специальная калибровка эхолота Simrad EK15 проводилась по искусственному ГФ. Основу искусственного ГФ [11] составил генератор пузырьков, состоящий из последовательно соединенных баллона с азотом, системы подачи газа, позволяющей задавать нужный поток газа, и опускаемого в водо- ем сопла с выходным диаметром 3 мм. Потоки искусственного ГФ задавались в диапазоне от 0,02 до $1,27 \pi / \mathrm{c}$.

Калибровка заключалась в получении эмпирической зависимости уровня обратного рассеяния на глубине $\sim 39$ м от величины потока газа, выходящего из сопла, установленного на глубине 40 м и находящегося в центре диаграммы направленности эхолота Simrad EK15. Для калибровки эхолота применялся газ азот. Как показано в работе [25], для калибровки эхолота по искусственному факелу вместо метана можно использовать любой другой газ, в том числе, более безопасный, инертный газ - азот. Диаметр выходящих из сопла пузырьков, определенных по данным прямых оптических наблюдений, лежал в диапазоне 3-12 мм, что соответствует характерным размерам естественных газовых пузырьков в зонах пузырьковой разгрузки $[10,24,26,45]$. При таких размерах пузырьков рабочая частота эхолота 200 кГц много выше резонансной частоты пузырьков, что удовлетворяет условию, необходимому для проведения калибровки. На рис. 4 представлена эхограмма, полученная во время калибровки эхолота, когда поток вытекающего из сопла газа составлял 1,27 л/с. На рисунке отчетливо выделяется дно и расположенная выше него область повышенного рассеяния, связанная с всплывающими пузырьками. Выше глубин $\sim 15$ м акустический сигнал от всплывающих пузырьков уменьшается, что связано с сильным приповерхностным течением, которое выносило всплывающие пузырьки из диаграммы направленности акустического преобразователя. 


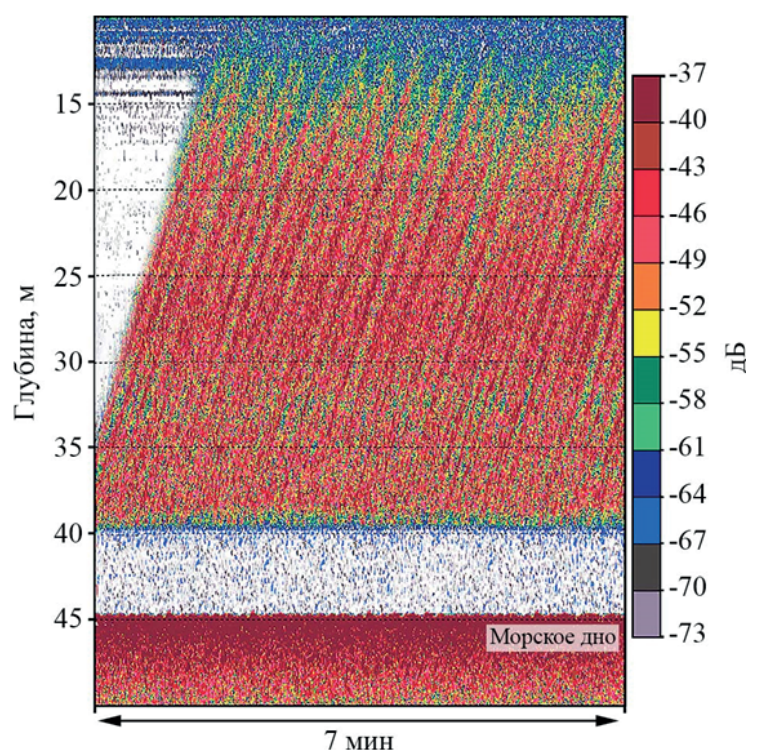

Рис. 4. Эхограмма, полученная во время калибровки научного эхолота Simrad EK15 по искусственному ГФ

Fig. 4. Hydro-acoustical image of artificial seep detected during simrad EK15 echosounder calibration

По результатам калибровочного эксперимента была построена кривая зависимости величины квадрата приведенного напряжения на выходе эхолота от потока газа, приведенная на рис. 5 . Каждая точка построена путем усреднения по 420 импульсам излучения по интервалу времени 7 мин.

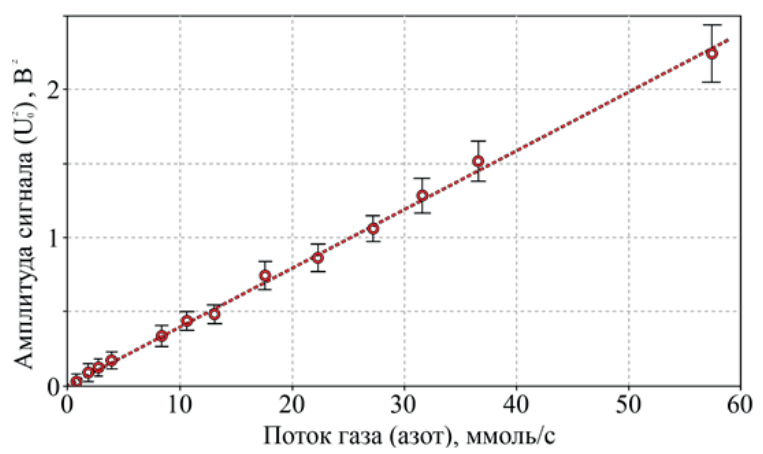

Рис. 5. График зависимости квадрата приведенной амплитуды сигнала на выходе эхолота от потока газа, переносимого всплывающими пузырьками в искусственНОМГФ

Fig. 5. Diagram of the amplitude of echo-signal dependence on calibration gas flow carried by arising bubbles

Видно, что экспериментальные точки хорошо ложатся на прямую линию, проходящую через начало координат, что говорит о существовании в используемом диапазоне потоков (1-57 ммоль/с) линейной зависимости между квадратом приведенного напряжения на выходе эхолота $U_{0}^{2}$ и потоком $F$. Это подтверждает обоснованность используемого выражений (8) и (9) для широкого диапазона потоков газа. Таким образом, метод, основанный на экспериментальном определении коэффициента $K$ в условиях, максимально приближенных к усло- виям натурных измерений, позволяет производить экспресс оценки потока метана, переносимого всплывающими пузырьками, без использования данных о пузырьках в ГФ и характеристиках применяемого гидроакустического оборудования. Отметим, что представленный способ калибровки может быть применен и для многолучевых эхолотов [25].

Сопоставление методов дистанционного определения потоков метана по измерению сечения обратного рассеяния от всплывающих в ГФ пузырьков и с помощью калибровки эхолота по искусственному ГФ проводилось в районе обширной области пузырьковой разгрузки метана на шельфе моря Лаптевых [10]. На рис. 6 представлен пример эхограммы, полученной с помощью научного эхолота Simrad EK15 во время пересечения обширной области пузырьковой разгрузки.

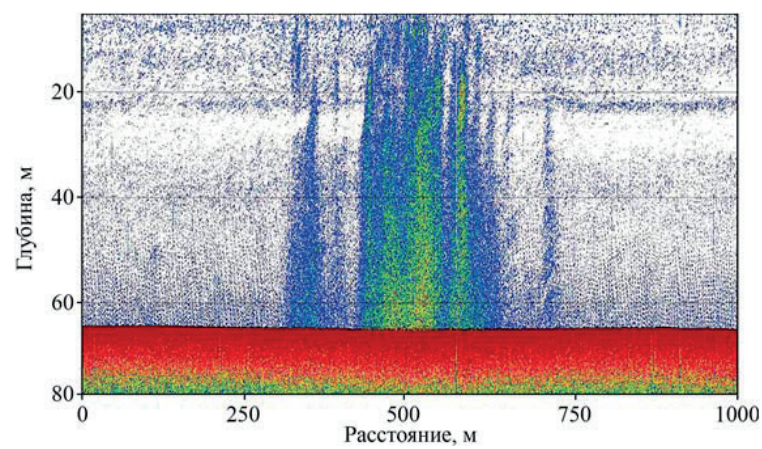

Рис. 6. Эхограмма пересечения обширной области пузырьковой разгрузки на шельфе м. Лаптевых

Fig. 6. Hydro-acoustical images of Laptev sea large seep

Поток метана из области пузырьковой разгрузки (рис 6), определенный дистанционным акустическим методом по данным измерения сечения обратного рассеяния от всплывающих пузырьков и расчета с помощью выражения (4) (с учетом экспериментально измеренных радиуса пузырьков и скорости всплытия) составил $0,27 \pm 0,06$ ммоль $\cdot \mathrm{M}^{-2} \cdot \mathrm{c}^{-1}$. Поток метана из той же области, но оцененный дистанционным акустическим методом по выражению (9) с помощью эхолота, откалиброванного по искусственному ГФ, составил $0,33 \pm 0,07$ ммоль $\cdot \mathrm{M}^{-2} \cdot \mathrm{c}^{-1}$. Отличие между двумя используемыми методами измерения потока метана составило 19 \%, при этом эти оценки между собой достоверно не отличаются $(p<0,05)$.

\section{Заключение}

В работе представлен обзор современных подходов для оценки потоков метана в водной толще, связанных с выходящими из дна и всплывающими пузырьками, акустическими дистанционными методами. На примере обширной области пузырьковой разгрузки $\mathrm{CH}_{4}$ на шельфе моря Лаптевых обоснована репрезентативность предложенного нового метода, основанного на расчете по сечению обратного рассеяния всплывающих пузырьков $\mathrm{CH}_{4}$. Показано, что оценки величины пузырькового потока, полученные двумя методами: 1) новым мето- 
дом, разработанным авторами, 2) методом калибровки эхолота по искусственному газовому факелу дают схожие результаты: $0,27 \pm 0,06$ и $0,33 \pm 0,07$ ммоль $\cdot \mathrm{M}^{-2} \cdot \mathrm{c}^{-1}$, соответственно. Таким образом, на практике для дистанционной и оперативной оценки потоков $\mathrm{CH}_{4}$ с участков его пузырьковой разгрузки можно использовать оба метода, с

\section{СПИСОК ЛИТЕРАТУРЫ}

1. Loncke L., Mascle J. Mud volcanoes, gas chimneys, pockmarks and mounds in the Nile deep-sea fan (Eastern Mediterranean): geophysical evidences // Marine and Petroleum Geology. 2004. - № 21. - P. 669-689.

2. Fate of rising methane bubbles in stratified waters: How much methane reaches the atmosphere? / D.F. McGinnis, J. Greinert, Y. Artemov, S.E. Beaubien, A. Wuest // Journal of Geophysical Research. - 2006. - № 111. - P. 9007-9017.

3. Milkov A.V. Worldwide distribution of submarine mud volcanoes and associated gas hydrates // Marine Geology. - 2000. № 167. - P. 29-42.

4. Саломатин А.С., Юсупов В.И. Акустические исследования газовых «Факелов» Охотского моря // Океанология. - 2011. № 51. - С. 857-865.

5. Деградация подводной мерзлоты и разрушение гидратов шельфа морей Восточной Арктики как возможная причина «метановой катастрофы»: некоторые результаты комплексных исследований 2011 года / В.И. Сергиенко, Л.И. Лобковский, И.П. Семилетов, О.В. Дударев, Н.Н. Дмитревский, Н.Е. Шахова, Н.Н. Романовский, Д.А. Космач, Д.Н. Никольский, С.Л. Никифоров, А.С. Саломатин, Р.А. Ананьев, А.Г. Росляков, А.Н. Салюк, В.Н. Карнаух, Д.Б. Черных, В.Е. Тумской, В.И. Юсупов, А.В. Куриленко, Е.М. Чувилин, Б.А. Буханов // Доклады Академии наук. - 2012. - № 3. - С. 330-335.

6. Postglacial response of Arctic Ocean gas hydrates to climatic amelioration / P. Serov, S. Vadakkepuliyambatta, J. Mienert, H. Patton, A. Portnov, A. Silyakova, G. Panieri, M. Carroll, J. Carroll, K. Andreassen, A. Hubbard // Proceedings of the National Academy of Sciences. - 2017. - № 114 (24). - P. 6215-6220.

7. Zhang M., Qiao F., Song Z. Observation of atmospheric methane in the Arctic Ocean up to $87^{\circ}$ north // Science China. - 2016. № 60. - P. 173-179.

8. Extensive methane venting to the atmosphere from sediments of the East Siberian Arctic Shelf / N. Shakhova, I. Semiletov, A. Salyuk, V. Iossoupov, D. Kosmach, 0. Gustafsson // Science. 2010. - № 327. - P. 1246-1249.

9. Methane in Water and Bottom Sediments in Three Sections in the Kara and Laptev Seas / A.A. Vetrov, N.V. Lobus, A.N. Drozdova, A.N. Belyaev, E.A. Romankevich // Oceanology. - 2018. № 58. - P. 198-204.

10. Massive blow-out craters formed by hydrate-controlled methane expulsion from the Arctic seafloor / K. Andreassen, A. Hubbard, M. Winsborrow, H. Patton, S. Vadakkepuliyambatta, A. PlazaFaverola, E. Gudlaugsson, P. Serov, A. Deryabin, R. Mattingsdal, J. Mienert, S. Bünz // Science. - 2017. - № 356 (6341). P. 948-953.

11. The East Siberian Arctic Shelf: towards further assessment of permafrost-related methane fluxes and role of sea ice N. Shakhova, I. Semiletov, V. Sergienko, L. Lobkovsky, V. Yusupov, A. Salyuk, A. Salomatin, D. Chernykh, D. Kosmach, G. Panteleev, D. Nicolsky, V. Samarkin, S. Joye, A. Charkin, 0. Dudarev, A. Meluzov, 0. Gustafsson // Philosophical Transactions of the Royal Society A. - 2015. - № 373. - P. 1-13.

12. Methane Feedbacks to the Global Climate System in a Warmer World / J. Dean, J. Middelburg, T. Röckmann, R. Aerts, L. Blauw, M. Egger, M. Jetten, A. de Jong, 0. Meisel, 0. Rasi- учетом занижения расчета потока по сечению обратного рассеяния примерно на $20 \%$ относительно реальных значений.

Работа выполнена при частичной поддержке проекта Российского Научного Фонда, РНФ (№ 15-17-20032 НШ ), проекта Правительства Российской Федерации (проект № 14.Z50.31.0012 ИС) и проекта РНФ (№ 18-77-10004 ДЧ).

graf, C. Slomp, M. H. in't Zandt, A. Dolman // Reviews of Geophysics. - 2018. - № 56 (1). - P. 1-44.

13. Whiteman G., Hope C., Wadhams P. Climate science: Vast costs of Arctic change // Nature. - 2013. - № 499. - P. 401-403.

14. Nicolsky D., Shakhova N. Modeling sub-sea permafrost in the East Siberian Arctic Shelf: the Dmitry Laptev Strait // Environmental Research Letters. - 2010. - № 5 (1). - P. 1-10.

15. Current rates and mechanisms of subsea permafrost degradation in the East Siberian Arctic Shelf / N. Shakhova, I. Semiletov, 0. Gustafsson, V. Sergienko, L. Lobkovsky, 0. Dudarev, V. Tumskoy, M. Grigoriev, A. Mazurov, A. Salyuk, R. Ananiev, A. Koshurnikov, D. Kosmach, A. Charkin, N. Dmitrevsky, V. Karnaukh, A. Gunar, A. Meluzov, D. Chernykh // Nature Communications. - 2017. - № 8. - P. 1-13.

16. Shakhova N.E., Alexeev V.A., Semiletov I.P. Predicting methane emission on the East-Siberian Shelf // Doklady Earth Sciences. 2009. - № 430 (2). - P. 190-193.

17. Judd A.G. The global importance and context of methane escape from the seabed // Geo-Marine Letters. - 2003. - № 23. P. $147-154$.

18. Mazurenko L.L., Soloviev V.A. Worldwide distribution of deepwater fluid venting and potential occurrences of gas hydrate accumulations // Geo-Marine Letters. - 2003. - № 23. P. $162-176$.

19. Lelieveld J., Crutzen P.J., Dentener F.J. Changing concentration, lifetime and climate forcing of atmospheric methane // Tellus. - 1998. - № 50. - P. 128-150.

20. 1300 -m-high rising bubbles from mud volcanoes at $2080 \mathrm{~m}$ in the Black Sea: Hydroacoustic characteristics and temporal variability / J. Greinert, Y. Artemov, V. Egorov, M. de Batist, D. McGinnisd // Earth and Planetary Science Letters. - 2006. - № 244. - P. 1-15.

21. Greinert J., Nützel B. Hydroacoustic experiments to establish a method for the determination of methane bubble fluxes at cold seeps // Geo-Marine Letters. - 2004. - № 24. - P. 75-85.

22. Effects of climate change on methane emissions from seafloor sediments in the Arctic Ocean: a review / R. James, P. Bousquet, I. Bussmann, M. Haeckel, R. Kipfer, I. Leifer, H. Niemann, I. Ostrovsky, J. Piskozub, G. Rehder, T. Treude, L. Vielstadte, J. Greinert // Limnology and oceanography. - 2016. - № 62. P. 283-299.

23. Leifer I., Culling D. Formation of seep bubble plumes in the Coal Oil Point seep field // Geo-Marine Letters. - 2010. - № 30. P. 339-353.

24. Leifer I., Patro R.K. The bubble mechanism for methane transport from the shallow sea bed to the 838 surface: a review and sensitivity study // Continental Shelf Research. - 2002. № 22. - P. 2409-2428.

25. Sonar Gas Flux Estimation by Bubble Insonification: Application to Methane Bubble Fluxes from the East Siberian Arctic Shelf Seabed / I. Leifer, D. Chernykh, N. Shakhova, I. Semiletov // The Cryosphere. - 2017. - № 11. - P. 1333-1350.

26. Муякшин С.И., Заутер Э. Дистанционный акустический метод определения производительности подводного источника газовых пузырьков / Океанология. - 2010. - № 6. - С. 1045-1051.

27. Nikolovska A., Sahling H., Bohrman G. Hydroacoustic methodology for detection, localization and quantification of gas bubbles rising from the seafloor at gas seeps from the eastern Black Sea // 
Geochemistry. Geophysics. Geosystems. - 2008. - № 9. P. 1-10.

28. Акустическая оценка концентрации метана в водной толще в областях его пузырьковой разгрузки / А.С. Саломатин, В.И. Юсупов, 0.Ф. Верещагина, Д.В. Черных // Акустический журнал. - 2014. - № 5. - С. 638-644.

29. A new methodology for quantifying bubble flow rates in deep water using splitbeam echosounders: Examples from the Arctic offshore NW Svalbard / M. Veloso, J. Greinert, J. Mienert, M. de Batist // Limnology and Oceanography: methods. - 2015. № 13. - P. 267-287.

30. Medwin H., Clay C.S. Fundamentals of Acoustical Oceanography. - San Diego: Academic Press Books, 1998. - 712 p.

31. Urick R.J. Principles of Underwater Sound for Engineers. - New York: McGraw-Hill, 1967. 342 p.

32. Artemov Y.G. Software support for investigation of natural methane seeps by hydroacoustic method // Морський екологічний журнал. - 2006. - № 5. - Р. 57-71.

33. Methane seeps in the Black Sea: discovery, quantification and environmental assessment / V.N. Egorov, Y.G. Artemov, S.B. Gulin, G.G. Polikarpov // Journal of the Black Sea/Mediterranean Environment. - 2011. - № 17. - P. 171-185.

34. Thermal anomalies associated with shallow gas hydrates in the K-2 mud volcano, Lake Baikal / J. Poort, 0.M. Khlystov, L. Naudts, A.D. Duchkov, H. Shoji, S. Nishio, M. Batist, A. Hachikubo, M. Kida, H. Minami, A.Y. Manakov, M.V. Kulikova, A.A. Krylov // Geo-Marine Letters. - 2012. - V. 32. - № 5. P. 407-417.

35. Jerram K., Weber T., Beaudoin J. Split-beam echo sounder observations of natural methane seep variability in the northern Gulf of Mexico // Geochemistry. Geophysics. Geosystems. - 2015. № 16 (3). - P. 736-750.

36. Estimation of methane fluxes from bottom sediments of Lake Baikal / N.G. Granin, S.I. Muyakshin, M.M. Makarov, K.M. Kucher, A.A. Granina, I.B. Mizandrontsev // Geo-Marine Letters. 2012. - № 32. - P. 427-436.

37. Munk W., Wunsch C. Ocean acoustic tomography: a scheme for large scale monitoring // Deep-Sea Research. - 1978. - № 26. P. 123-161.

38. Seepage from an arctic shallow marine gas hydrate reservoir is insensitive to momentary ocean warming / W. Hong, M. Torres, J. Carroll, A. Crémière, G. Panieri, H. Yao, P. Serov // Nature Communications. - 2017. - № 8. - P. 1-14.
39. Maini B. B., Bishnoi P. R. Experimental investigation of hydrate formation behaviour of a natural gas bubble in a simulated deep sea environment // Chemical Engineering Science. - 1981. № 36. - P. 183-189.

40. Ishimaru A. Wave Propagation and Scattering in Random Media. - New York: Wiley, 1999. - $600 \mathrm{p}$.

41. Maksimov A.0., Yusupov V.I. Coupled oscillations of a pair of closely spaced bubbles // European Journal of MechanicsB/Fluids. - 2016. - № 60. - P. 164-174.

42. Acoustic estimates of methane gas flux from the seabed in a $6000 \mathrm{~km}^{2}$ region of the Northern Gulf of Mexico / T.C. Weber, L.A. Mayer, K. Jerram, J. Beaudoin, Y. Rzhanov, D. Lavalvo // Geochemistry. Geophysics. Geosystems. - 2014. - V. 15. P. 1911-1925.

43. Distribution and height of methane bubble plumes on the Cascadia Margin characterized by acoustic imaging / K.U. Heeschen, A.M. Tre'hu, R.W. Collier, E. Suess, G. Rehder // Geophysical Research Letters. - 2003. - № 12. - P. 45-49.

44. Hornafius J.S., Quigley D., Luyendyk B.P. The world's most spectacular marine hydrocarbon seeps (Coal Oil Point, Santa Barbara Channel, California): Quantification of emissios // Journal of Geophysical Research. - 1999. - № 9. - P. 20703-20711.

45. Distributed natural gas venting offshore along theCascadia margin / M. Riedel, M. Scherwath, M. Römer, M. Veloso, M. Heesemann, G.D. Spence // Nature Communications. - 2018. - V. 9. № 1. - P. 1-14.

46. Ainslie M.A., Leighton T.G. Review of scattering and extinction cross-sections, damping factors, and resonance frequencies of a spherical gas bubble // Journal of acoustical society of America. - 2010, - № 130. - P. 3184-3208.

47. Improved calibration of hydroacoustic equipment with copper spheres / K.G. Foote, H.P. Knudsen, G. Vestnes, R. Brede, L. Nielsen // ICES CM. - 1981. - № 18. - P. 8-20.

48. MacLennan D.N. The theory of solid spheres as sonar calibration targets // Scottish Fisheries Research. - 1981. - № 22. P. 1-17.

49. MacLennan D.N., Dunn J.R. Estimation of sound velocities from resonance measurements on tungsten carbide calibration spheres // Journal of Sound and Vibration. - 1984. - № 97. - P. 231-331.

Поступила 10.08.2018 г.

\section{Информация об авторах}

Черных Д.В., кандидат технических наук, научный сотрудник Лаборатории акустической океанографии Тихоокеанского океанологического института им. В.И. Ильичева; сотрудник Международной научно-образовательной лаборатории изучения углерода арктических морей Национального исследовательского Томского политехнического университета.

Юсупов В.И., кандидат физико-математических наук, научный сотрудник Лаборатории лазерной химии Института фотонных технологий ФНИЦ «Кристаллография и фотоника» РАН.

Саломатин A.C., кандидат физико-математических наук, заведующий Лабораторией акустической океанографии Тихоокеанского океанологического института им. В.И. Ильичева.

Космач Д.А., научный сотрудник Лаборатории арктических исследований Тихоокеанского океанологического института им. В.И. Ильичева; сотрудник Международной научно-образовательной лаборатории изучения углерода арктических морей Национального исследовательского Томского политехнического университета.

Константинов A.B., студент кафедры приборостроения инженерной школы Дальневосточного федерального университета.

Силионов В.И., младший научный сотрудник Лаборатории физики живой материи Центра киральной биофотоники Департамента фармации и фармакологии Школы биомедицины Дальневосточного федерального университета. 
Мазуров A.к., доктор геолого-минералогических наук, профессор отделения геологии Инженерной школы природных ресурсов Национального исследовательского Томского политехнического университета.

Салюк A.Н., кандидат физико-математических наук, ведущий научный сотрудник Лаборатории арктических исследований Тихоокеанского океанологического института им. В.И. Ильичева.

Шахова H.E., доктор геолого-минералогических наук, профессор, ведущий научный сотрудник Международной научно-образовательной лаборатории изучения углерода арктических морей Национального исследовательского Томского политехнического университета.

Густафсон $0 ., \mathrm{PhD}$, профессор кафедры наук об окружающей среде и аналитической химии Центра исследований климата им. Б. Болина Стокгольмского университета.

Колюбакин A.A., научный сотрудник Центра морских исследований МГУ им. М.В. Ломоносова; ООО «Арктический научный центр» .

Гершелис $\boldsymbol{E}$.B., ассистент отделения геологии Инженерной школы природных ресурсов Национального исследовательского Томского политехнического университета.

Лобковский Л.И., член-корреспондент Российской Академии наук (РАН), доктор физико-математических наук, заведующий Лабораторией геодинамики, георесурсов, георисков и геоэкологии Института океанологии им. П.П. Ширшова РАН.

Селилетов И.П., член-корреспондент РАН, доктор географических наук, заведующий Лабораторией арктических исследований Тихоокеанского океанологического института им. В.И. Ильичева; научный руководитель Международной научно-образовательной лаборатории изучения углерода арктических морей Национального исследовательского Томского политехнического университета. 


\section{NEW ACOUSTICAL TECHNIQUE TO QUANTIFY METHANE EBULLITION IN SEDIMENT WATER COLUMN: A CASE STUDY IN THE LAPTEV SEA, THE ARCTIC OCEAN}

Denis V. Chernykh, ${ }^{1,2}$ denis.chernykh.vl@gmail.com

Vladimir I. Yusupov ${ }^{3}$, iouss@yandex.ru

\section{Alexander S. Salomatin',} salomatin@poi.dvo.ru

\section{Denis A. Kosmach ${ }^{1,2}$,} den-kosmach@mail.ru

\section{Anton V. Konstantinov ${ }^{4}$,} toshic.konstantinov@gmail.com

\section{Vyacheslav I. Silionov ${ }^{4}$,} truexenox@gmail.com

\section{Alexey K. Mazurov², akm@tpu.ru}

\author{
Anatoly N. Salyuk', \\ san@poi.dvo.ru
}

\author{
Natalia E. Shakhova ${ }^{2}$, \\ NataliaFletcher@yahoo.com \\ Orjan Gustafsson ${ }^{5}$, \\ Orjan.Gustafsson@aces.su.se
}

\author{
Andrey A. Kolyubakin ${ }^{6,7}$, \\ andrey.kolyubakin@gmail.com
}

\author{
Elena V. Gershelis', \\ elenapanova@tpu.ru
}

\section{Leopold I. Lobkovsky8, llobkovsky@ocean.ru}

Igor P. Semiletov ${ }^{1,2}$ ipsemiletov@alaska.edu; igorsm@poi.dvo.ru

\footnotetext{
1 Russian Academy of Sciences, Pacific Oceanological Institute, 43, Baltiyiskaya street, Vladivostok, 690041, Russia.

${ }^{2}$ National Research Tomsk Polytechnic University, 30, Lenin avenue, Tomsk, Russia.

${ }^{3}$ Russian Academy of Sciences, Research Center "Crystallography and Photonics», 59, Leninsky avenue, Moscow, 119333, Russia.

${ }^{4}$ Far Eastern Federal University, 8, Sukhanov street, Vladivostok, 690090, Russia.

${ }^{5}$ Stockholm University, 8, Svante Arrhenius vag, Stockholm, 10691, Sweden.

${ }^{6}$ Lomonosov Moscow State University, 1, Leninskiye Gory, Moscow, 119991, Russia.

${ }^{7}$ Arctic Research Centre, 155, Leninsky Prospect, Moscow, 119333, Russia.

${ }^{8}$ Institute of Oceanology im. P. Shirshov of the Russian Academy of Sciences, 36, Nahimovskiy avenue, Moscow, 117997, Russia.
}

\footnotetext{
The relevance of the research is caused by the need to develop a scientifically based approach to quantitative estimation of bubble transfer of methane and other gases based on acoustic techniques, which allow reliable estimate of methane flow from the bubble unloading areas by sound locators and submarine sonars.

The main aim of the research is to investigate the possible application of an acoustical technique based on acoustic scattering in bubble plumes vs the acoustical technique based on calibration which was applied to quantify in situ sonar observations; to show that both techniques can be used for a quantification of methane ebullition in the bottom-water column system.

Objects: gas flares or seeps - the emanations of gas in the form of rising bubbles from the seabottom, which form stable regions of their increased concentration in the water column.

Methods: modification of acoustical techniques based on acoustic scattering in bubble plumes and on ist calibration which was applied by authors to quantify in situ single sonar observations.

Results. We demonstrate a first attempt to use acoustical techniques based on (1) acoustic scattering in bubble plumes vs acoustical technique based on (2) calibration which was applied to quantify in situ sonar observations. It has been shown that both techniques can be used for a quatitative express-evaluation of methane ebullition in the bottom-water system in any aquatic ecosystem including seas, lakes, and rivers, while the first acoustical technique gives the bubble efflux values $\sim 20 \%$ lower then the second acoustical technique.
} 
Key words:

Arctic, acoustic estimation, methane, seep, Laptev sea, bubbles transport, methane flux.

The research was partially supported by the project of the Russian Science Foundation (no.15-17-20032 NSh). Some aspects of the research were supported by the project of the Government of the Russian Federation (project no. 14.Z50.31.0012 IS) and project RSF (no. 18-77-10004 DCh).

\section{REFERENCES}

1. Loncke L., Mascle J. Mud volcanoes, gas chimneys, pockmarks and mounds in the Nile deep-sea fan (Eastern Mediterranean): geophysical evidences. Marine and Petroleum Geology, 2004, no. 21 , pp. 669-689.

2. McGinnis D.F., Greinert J., Artemov Y., Beaubien S.E., Wuest A. Fate of rising methane bubbles in stratified waters: How much methane reaches the atmosphere? Journal of Geophysical Research, 2006, no. 111, pp. 9007-9017.

3. Milkov A.V. Worldwide distribution of submarine mud volcanoes and associated gas hydrates. Marine Geology, 2000, no. 167, pp. 29-42.

4. Salomatin A.S., Yusupov V.I. Akusticheskie issledovaniya gazovykh «Fakelov» Okhotskogo moray [Acoustic Investigations of Gas «Flares» in the Sea of Okhotsk]. Oceanology, 2011, no. 51, pp. 857-865.

5. Sergienko V.I., Lobkovskii L.I., Semiletov I.P., Dudarev 0.V., Dmitrievskii N.N., Shakhova N.E., Romanovskii N.N., Kosmach D.A., Nikolskii, D.N., Nikiforov S.L., Salomatin A.S., Ananev R.A., Roslyakov A.G., Salyuk A.N., Karnaukh V.V., Chernykh D.B., Tumskoi V.E., Yusupov V.I., Kurilenko A.V., Chuvilin E.M., Bukhanov B.A. Degradatsiya podvodnoy merzloty i razrushenie gidratov shelfa morey Vostochnoy Arktiki kak vozmozhnaya prichina «metanovoy katastrofy»: nekotorye rezultaty kompleksnykh issledovany 2011 goda [Degradation of submarine permafrost and destruction of hydrates on the shelf of east arctic seas as a potential cause of the Methane Catastrophe: some results of integrated studies in 2011]. Doklady Earth Sciences, 2012, no. 446, pp. 1132-1137.

6. Serov P., Vadakkepuliyambatta S., Mienert J., Patton H., Portnov A., Silyakova A., Panieri G., Carroll M., Carroll J., Andreassen K., Hubbard A. Postglacial response of Arctic Ocean gas hydrates to climatic amelioration. Proc. of the National Academy of Sciences, 2017, no. 114 (24), pp. 6215-6220.

7. Zhang M., Qiao F., Song Z. Observation of atmospheric methane in the Arctic Ocean up to $87^{\circ}$ north. Science China, 2016, no. 60, pp. 173-179.

8. Shakhova N., Semiletov I., Salyuk A., Iossoupov V., Kosmach D., Gustafsson 0. Extensive methane venting to the atmosphere from sediments of the East Siberian Arctic Shelf. Science, 2010, no. 327 , pp. 1246-1249.

9. Vetrov A.A., Lobus N.V., Drozdova A.N., Belyaev A.N., Romankevich E.A. Methane in Water and Bottom Sediments in Three Sections in the Kara and Laptev Seas. Oceanology, 2018, no. 58, pp. 198-204.

10. Andreassen K., Hubbard A., Winsborrow M., Patton H., Vadakkepuliyambatta S., Plaza-Faverola A., Gudlaugsson E., Serov P., Deryabin A., Mattingsdal R., Mienert J., Bünz S. Massive blowout craters formed by hydrate-controlled methane expulsion from the Arctic seafloor. Science, 2017, no. 356 (6341), pp. 948-953.

11. Shakhova N., Semiletov, I., Sergienko, V., Lobkovsky, L., Yusupov, V., Salyuk, A., Salomatin, A., Chernykh, D., Kosmach, D., Panteleev, G., Nicolsky, D., Samarkin, V., Joye, S., Charkin, A., Dudarev, 0., Meluzov, A., Gustafsson 0. The East Siberian Arctic Shelf: towards further assessment of permafrost-related methane fluxes and role of sea ice. Philosophical Transactions of the Royal Society A, 2015, no. 373, pp. 1-13.

12. Dean J., Middelburg J., Röckmann T., Aerts R., Blauw L., Egger M., Jetten M., de Jong A., Meisel 0., Rasigraf 0., Slomp C., H. in’t Zandt M., Dolman A. Methane Feedbacks to the Global Climate System in a Warmer World Reviews of Geophysics, 2018, no. 56 (1), pp. 1-44.

13. Whiteman G., Hope C., Wadhams P. Climate science: Vast costs of Arctic change. Nature, 2013, no. 499, pp. 401-403.
14. Nicolsky D., Shakhova N. Modeling sub-sea permafrost in the East Siberian Arctic Shelf: the Dmitry Laptev Strait. Environmental Research Letters, 2010, no. 5 (1), pp. 1-10.

15. Shakhova N., Semiletov I., Gustafsson O., Sergienko V., Lobkovsky L., Dudarev 0., Tumskoy V., Grigoriev M., Mazurov A., Salyuk A., Ananiev R., Koshurnikov A., Kosmach D., Charkin A., Dmitrevsky N., Karnaukh V., Gunar A., Meluzov A., Chernykh D. Current rates and mechanisms of subsea permafrost degradation in the East Siberian Arctic Shelf. Nature Communications, 2017, no. 8, pp. 1-13.

16. Shakhova N.E., Alexeev V.A., Semiletov I.P. Predicting methane emission on the East-Siberian Shelf. Doklady Earth Sciences, 2009, no. 430 (2), pp. 190-193.

17. Judd A.G. The global importance and context of methane escape from the seabed. Geo-Marine Letters, 2003, no. 23, pp. 147-154.

18. Mazurenko L.L., Soloviev V.A. Worldwide distribution of deepwater fluid venting and potential occurrences of gas hydrate accumulations. Geo-Marine Letters, 2003, no. 23, pp. 162-176.

19. Lelieveld J., Crutzen P.J., Dentener F.J. Changing concentration, lifetime and climate forcing of atmospheric methane. Tellus, 1998, no. 50, pp. 128-150.

20. Greinert J., Artemov Y., Egorov V., De Batist M., McGinnisd D. 1300 -m-high rising bubbles from mud volcanoes at $2080 \mathrm{~m}$ in the Black Sea: Hydroacoustic characteristics and temporal variability. Earth and Planetary Science Letters, 2006, no. 244, pp. 1-15.

21. Greinert J., Nützel B. Hydroacoustic experiments to establish a method for the determination of methane bubble fluxes at cold seeps. Geo-Marine Letters, 2004, no. 24, pp. 75-85.

22. James R., Bousquet P., Bussmann I., Haeckel M., Kipfer R., Leifer I., Niemann H., Ostrovsky I., Piskozub J., Rehder G., Treude T., Vielstadte L., Greinert J. Effects of climate change on methane emissions from seafloor sediments in the Arctic Ocean: a review. Limnology and oceanography, 2016, no. 62, pp. 283-299.

23. Leifer I., Culling D. Formation of seep bubble plumes in the Coal Oil Point seep field. Geo-Marine Letters, 2010, no. 30, pp. 339-353.

24. Leifer I., Patro R.K. The bubble mechanism for methane transport from the shallow sea bed to the 838 surface: a review and sensitivity study. Continental Shelf Research, 2002, no. 22, pp. 2409-2428.

25. Leifer I., Chernykh D., Shakhova N., Semiletov I. Sonar Gas Flux Estimation by Bubble Insonification: Application to Methane Bubble Fluxes from the East Siberian Arctic Shelf Seabed. The Cryosphere, 2017, no. 11, pp. 1333-1350.

26. Muyakshin S.I., Sauter E. Distantsionny akustichesky metod opredeleniya proizvoditelnosti podvodnogo istochnika gazovykh puzyrkov [Hydroacoustic Method for the Quantification of the Gas Flux from a Submersed Bubble Plume]. Oceanology, 2010, no. 50 , pp. $995-1001$.

27. Nikolovska A., Sahling H., Bohrman G. Hydroacoustic methodology for detection, localization and quantification of gas bubbles rising from the seafloor at gas seeps from the eastern Black Sea. Geochemistry. Geophysics. Geosystems, 2008, no. 9, pp. 1-10.

28. Salomatin A.S., Yusupov V.I., Vereshchagina O.F., Chernykh D.V. Akusticheskaya otsenka kontsentratsii metana v vodnoy tolshche $\mathrm{v}$ oblastyakh ego puzyrkovoy razgruzki [Acoustic estimate of methane concentration in a water column in regions of methane bubble release]. Acoustical Physics, 2014, no. 60, pp. 671-677. 
29. Veloso M., Greinert J., Mienert J., De Batist M. A new methodology for quantifying bubble flow rates in deep water using splitbeam echosounders: Examples from the Arctic offshore NW Svalbard. Limnology and Oceanography: methods, 2015, no. 13, pp. 267-287.

30. Medwin H., Clay C.S. Fundamentals of Acoustical Oceanography. San Diego, Academic Press Books, 1998. 712 p.

31. Urick R.J. Principles of Underwater Sound for Engineers. New York, McGraw-Hill, 1967. 342 p.

32. Artemov Y.G. Software support for investigation of natural methane seeps by hydroacoustic method. Morsky ekologichny zhurnal, 2006, no. 5, pp. 57-71.

33. Egorov V.N., Artemov Y.G., Gulin S.B., Polikarpov G.G. Methane seeps in the Black Sea: discovery, quantification and environmental assessment. Journal of the Black Sea/Mediterranean Environment, 2011, no. 17, pp. 171-185.

34. Poort J., Khlystov 0.M., Naudts L., Duchkov A.D., Shoji H., Nishio S., Batist M., Hachikubo A., Kida M., Minami H., Manakov A.Y., Kulikova M.V., Krylov A.A. Thermal anomalies associated with shallow gas hydrates in the K-2 mud volcano, Lake Baikal. Geo-Marine Letters, 2012, vol. 32, no. 5, pp. 407-417.

35. Jerram K., Weber T., Beaudoin J. Split-beam echo sounder observations of natural methane seep variability in the northern Gulf of Mexico. Geochemistry. Geophysics. Geosystems, 2015, no. $16(3)$, pp. $736-750$.

36. Granin N.G., Muyakshin S.I., Makarov M.M., Kucher K.M., Granina A.A., Mizandrontsev I.B. Estimation of methane fluxes from bottom sediments of Lake Baikal. Geo-Marine Letters, 2012, no. 32 , pp. 427-436.

37. Munk W., Wunsch C. Ocean acoustic tomography: a scheme for large scale monitoring. Deep-Sea Research, 1978, no. 26, pp. 123-161.

38. Hong W., Torres M., Carroll J., Crémière A., Panieri G., Yao H., Serov P. Seepage from an arctic shallow marine gas hydrate reservoir is insensitive to momentary ocean warming. Nature Communications, 2017, no. 8, pp. 1-14.

39. Maini B. B., Bishnoi P. R. Experimental investigation of hydrate formation behaviour of a natural gas bubble in a simulated deep sea environment. Chemical Engineering Science, 1981, no. 36, pp. $183-189$.

40. Ishimaru A. Wave Propagation and Scattering in Random Media. New York, Wiley, 1999. 600 p.

41. Maksimov A.0., Yusupov V.I. Coupled oscillations of a pair of closely spaced bubbles. European Journal of Mechanics-B/Fluids, 2016, no. 60, pp. 164-174

42. Weber T.C., Mayer L.A., Jerram K., Beaudoin J., Rzhanov Y., Lavalvo D. Acoustic estimates of methane gas flux from the seabed in a $6000 \mathrm{~km}^{2}$ region of the Northern Gulf of Mexico. Geochemistry. Geophysics. Geosystems, 2014, no. 15, pp. 1911-1925.

43. Heeschen K.U., Tre'hu A.M., Collier R.W., Suess E., Rehder G. Distribution and height of methane bubble plumes on the Cascadia Margin characterized by acoustic imaging. Geophysical Research Letters, 2003, no. 12, pp. 45-49.

44. Hornafius J.S., Quigley D., Luyendyk B.P. The world's most spectacular marine hydrocarbon seeps (Coal Oil Point, Santa Barbara Channel, California): Quantification of emissios. Journal of Geophysical Research, 1999, no. 9, pp. 20703-20711.

45. Riedel M., Scherwath M., Römer M., Veloso M., Heesemann M., Spence G.D. Distributed natural gas venting offshore along the Cascadia margin. Nature Communications, 2018, vol. 9, no. 1, pp. 1-14.

46. Ainslie M.A., Leighton T.G. Review of scattering and extinction cross-sections, damping factors, and resonance frequencies of a spherical gas bubble. Journal of acoustical society of America, 2010, no. 130, pp. 3184-3208.

47. Foote K.G., Knudsen H.P., Vestnes G., Brede R., Nielsen L. Improved calibration of hydroacoustic equipment with copper spheres. ICES CM, 1981, no. 18, pp. 8-20.

48. MacLennan D.N. The theory of solid spheres as sonar calibration targets. Scottish Fisheries Research, 1981, no. 22, pp. 1-17.

49. MacLennan D.N., Dunn J.R. Estimation of sound velocities from resonance measurements on tungsten carbide calibration spheres. Journal of Sound and Vibration, 1984, no. 97, pp. 231-331.

Received: 10 August 2018.

\section{Information about the authors}

Denis V. Chernykh, Cand. Sc., researcher, Russian Academy of Sciences, Pacific Oceanological Institute; researcher, Nationa Research Tomsk Polytechnic University.

Vladimir I. Yusupov, Cand. Sc., researcher, Russian Academy of Sciences, Research Center «Crystallography and Photonics».

Alexander S. Salomatin, Cand. Sc., Head of the Laboratory, Russian Academy of Sciences, Pacific Oceanological Institute.

Denis A. Kosmach, researcher, Russian Academy of Sciences, Pacific Oceanological Institute; researcher, National Research Tomsk Polytechnic University.

Anton V. Konstantinov, student, Far Eastern Federal University.

Vyacheslav I. Silionov, junior researcher, Far Eastern Federal University.

Alexey K. Mazurov, Dr. Sc., professor, National Research Tomsk Polytechnic University

Anatoly N. Salyuk, Cand. Sc., lead researcher, Russian Academy of Sciences, Pacific Oceanological Institute.

Natalia E. Shakhova, Dr. Sc., professor, lead researcher, National Research Tomsk Polytechnic University.

Orjan Gustafsson, PhD, professor, Stockholm University.

Andrey A. Kolyubakin, researcher, Lomonosov Moscow State University.

Elena V. Gershelis, teaching assistant, National Research Tomsk Polytechnic University.

Leopold I. Lobkovsky, corresponding member of the Russian Academy of Sciences, Dr. Sc., professor, head of the Laboratory, Institute of Oceanology im. P. Shirshov of the Russian Academy of Sciences.

Igor P. Semiletov, corresponding member of the Russian Academy of Sciences, Dr. Sc., professor, head of the Laboratory, Russian Academy of Sciences, Pacific Oceanological Institute; scientific leader, National Research Tomsk Polytechnic University. 